\title{
Component of Study Protocol
}

National Cancer Institute

\section{Source}

National Cancer Institute. Component of Study Protocol. NCI Thesaurus. Code C70705.

A component of a plan on which a study is based. 